\title{
Desenvolvimento de um banco de dados para mapeamento da dispersão do carvão do amendoim no Brasil
}

\begin{abstract}
Cláudia Dantas Procópio (iD
Mestre em Ciência da Computação - Analista A da Embrapa Algodão, Campina Grande/PB, claudia.procopio@embrapa.br.

Cristiane Santana Custódio dos Santos Avelino (D)

Bacharel em Informática - Analista A da Embrapa Algodão, Campina Grande/PB, cristiane.avelino@embrapa.br.
\end{abstract}

Marcel Norie Bergamin Sugimoto (D) Estudante de Engenharia de Alimentos, Bolsista PIBIC/CNPq/Embrapa - Centro Universitário de Jaguariúna (UniFAJ), Jaguariúna/SP, sugimoto.marcel@gmail.com.

Dartanhã José Soares (iD)

Doutor em Fitopatologia - Pesquisador da Embrapa Algodão, Campina Grande/PB, dartanha.soares@embrapa.br.

\section{RESUMO}

O carvão do amendoim, causado pelo fungo Thecaphora frezzii, foi relatado pela primeira vez na Argentina em meados da década de 1990, e desde 2015 tem sido considerada uma das principais doenças do amendoim. Uma vez que o fungo é principalmente disperso pelas sementes, e considerando que o Brasil regularmente importa sementes da Argentina, foi observada a necessidade de iniciar o monitoramento da presença desse fungo no Brasil, de modo a evitar que está doença venha a causar prejuízos aos produtores brasileiros. Dessa forma, o objetivo deste trabalho é apresentar o desenvolvimento do banco de dados que foi criado para organizar as informações relativas ao mapeamento das áreas de produção de amendoim do Brasil quanto a distribuição do agente causal do carvão do amendoim. Foi utilizado o Sistema Gerenciador de Bancos de Dados (SGBD) PostgreSQL com extensão espacial PostGIS. Para apresentação dos dados foi desenvolvido um sistema de informação Web, na linguagem de programação Java que, a partir do servidor de mapas GeoServer, mostra a distribuição das amostras analisadas por município. Diferentes cores foram usadas para permitir a rápida visualização dos municípios onde a presença do patógeno foi confirmada ou não. Além de permitir a visualização da distribuição das amostras pelos interessados, o desenvolvimento do presente banco de dados será útil em estudos futuros visando entender melhor a dinâmica de dispersão do patógeno.

Palavras-chave: Arachis hypogaea L.; Distribuição geográfica; Thecaphora frezzii.

\section{Development of a database to map the distribution of peanut smut in Brazil}

\author{
ABSTRACT
}

The peanut smut, caused by the fungus Thecaphora frezzii, was first reported in commercial fields in Argentina in the middle 1990's, and since 2015, it has been regarded one of the most important disease of peanut. Since this fungus is mainly spread by seed, and regarding that peanut growers in Brazil regularly import seeds from Argentina, it was observed the necessity to start to monitor the presence of this pathogen in Brazil, to avoid the serious losses reported in Argentina. Thus the aim of the present work is to present the development of a database created to organize the information regarding the monitoring of peanut smut pathogen in peanut commercial production areas in Brazil. The database manager system 
used was PostgreSQL with the PostGIS extension. A web interface, built using Java programming language with data inserted in GeoServer, shows the distribution of analyzed samples per municipality using a colored circumference with size proportional to the total number of samples. Different colors were used to allow a quick visualization of municipalities where the presence of the pathogen was confirmed or not. The present database and its web interface will not only allow both growers and phytossanitary authorities to visualize the pathogen distribution throughout the peanut growing areas, but will also provide useful information to a better understanding of its epidemiology.

Keywords: Arachis hypogaea L.; Geographic distribution; Thecaphora frezzii.

\title{
Desarrollo de una base de datos para mapear la distribución del carbón de maní en Brasil
}

\begin{abstract}
RESUMEN
El carbón de maní, causada por el hongo Thecaphora frezzii, fue reportado la primera vez en campos comerciales en Argentina en la década de 1990, y desde 2015, se ha considerado una de las enfermedades más importantes del maní. Dado que este hongo se propaga principalmente por semillas, y una vez que los productores de maní de Brasil regularmente importan semillas de Argentina, se observó la necesidad de comenzar a monitorear la presencia de este patógeno en Brasil, para evitar las graves pérdidas reportadas en Argentina. Por esta razón, el objetivo del presente trabajo es presentar el desarrollo de una base de datos creada para organizar la información sobre el monitoreo del carbón de maní en las áreas de producción comercial de maní en Brasil. El sistema de administrador de base de datos utilizado fue PostgreSQL con la extensión PostGIS. Una interfaz web, construida utilizando lenguaje de programación Java con datos insertados en GeoServer, muestra la distribución de las muestras analizadas por municipalidad utilizando una circunferencia con tamaño proporcional al número total de muestras. Se utilizaron diferentes colores para permitir una visualización rápida de las municipalidades donde se confirmó o no la presencia del patógeno. La base de datos y su interfaz web no solo permitirán a los productores y las autoridades fitosanitarias visualizar la distribución de patógenos en las áreas de cultivo de maní, sino que también proporcionarán información útil para una mejor comprensión de su epidemiología.
\end{abstract}

Palabras claves: Arachis hypogaea L.; Distribución geográfica; Thecaphora frezzii.

\section{Introdução}

O carvão do amendoim, doença causada pelo fungo Thecaphora frezzii, foi recentemente considerada a doença mais importante dessa cultura na Argentina (RAGO et al., 2017). Em virtude do seu agente causal ser transmitido pelas sementes, e considerando que o Brasil importa regularmente sementes oriundas da Argentina, existe um elevado risco de estar ocorrendo uma introdução sistemática deste patógeno nas áreas de produção de amendoim do Brasil. No entanto, a presença desse patógeno em áreas comerciais de amendoim do Brasil ainda não foi confirmada. Isso, porém, não significa dizer que o mesmo não está presente. Devido as 
características específicas deste patossistema, a constatação do patógeno, em nível de campo, normalmente só é possível quando o mesmo está amplamente disperso na área de produção e a intensidade da doença já atingiu níveis elevados (SOARES, 2019).

Em estágios iniciais de introdução e dispersão desse tipo de patógeno, somente é possível determinar a presença do mesmo por meio de estudos sistemáticos de amostragem. Dessa forma, desde a safra 2018/2019, a Embrapa tem conduzido um estudo para mapear as áreas de produção de amendoim do Brasil quanto a presença do agente causal do carvão do amendoim.

Devido a natureza do trabalho e a importância do patógeno para a cadeia produtiva do amendoim do Brasil, viu-se a necessidade de criar uma forma que permitisse o acompanhamento, por parte do setor produtivo e demais interessados, da distribuição das amostras analisadas e a identificação, em estágios iniciais, de potenciais focos de introdução do patógeno. Dessa forma, para organizar, e disponibilizar as informações referentes a este estudo foi desenvolvido um banco de dados, denominado de "Radar - Carvão do Amendoim", de forma a auxiliar não só o mapeamento de áreas livres ou não do patógeno, como também subsidiar estudos futuros visando entender melhor a dinâmica de dispersão e sobrevivência do patógeno.

\section{Material e métodos}

Levando-se em conta a biologia do patógeno e a epidemiologia da doença, foi preparado um formulário para ser preenchido com informações de cada amostra recebida (SOARES, 2018). A coleta, o preenchimento das informações e o envio das amostras foi feita tanto por auditores fiscais federais agropecuários, quanto, de forma voluntária, por produtores ou cooperativas de produtores de amendoim do estado de São Paulo (SUGIMOTO et al., 2019). Além das informações de origem geográfica da amostra (município, estado, nome da fazenda e coordenadas geográficas), foram solicitadas informações relativas ao tipo de solo, tipo de preparo de 
solo, sistema de cultivo, cultivo anterior, número de anos com cultivo sucessivo de amendoim, número de anos sem cultivo de amendoim, variedade, classe da semente, se fez correção do solo, se fez tratamento de sementes e o tamanho da gleba (Tabela 1). Os dados relativos a cada amostra e o resultado da análise quanto a presença de estruturas do patógeno foram organizados em um template (planilha excel) e posteriormente importados para o banco de dados.

Tabela 1. Exemplo de parte do conjunto de informações utilizadas para construir o banco de dados relativo ao "Radar - Carvão do Amendoim".

\begin{tabular}{|c|c|c|c|c|c|}
\hline $\begin{array}{c}\text { SOLO } \\
\text { PREDOMINANTE }\end{array}$ & $\begin{array}{c}\text { CULTIVO } \\
\text { ANTERIOR }\end{array}$ & SISTEMA DE CULTIVO & $\begin{array}{l}\text { TRATAMENTO } \\
\text { SEMENTE }\end{array}$ & $\begin{array}{c}\text { REALIZOU } \\
\text { ADUBAÇÃO? }\end{array}$ & $\begin{array}{l}\text { REALIZOU } \\
\text { CALAGEM? }\end{array}$ \\
\hline ARENOSO & $\begin{array}{l}\text { CANA-DE- } \\
\text { AÇÚCAR }\end{array}$ & CONVENCIONAL & $\begin{array}{c}\text { VITAVAX THIRAM } 200 \\
\text { SC }\end{array}$ & NÃO & SIM \\
\hline ARGILOSO & $\begin{array}{l}\text { CANA-DE- } \\
\text { AÇÚCAR }\end{array}$ & CONVENCIONAL & $\begin{array}{c}\text { VITAVAX THIRAM } 200 \\
\text { SC }\end{array}$ & NÃO & SIM \\
\hline ARENOSO & $\begin{array}{l}\text { CANA-DE- } \\
\text { AÇÚCAR }\end{array}$ & CONVENCIONAL & $\begin{array}{c}\text { VITAVAX THIRAM } 200 \\
\text { SC }\end{array}$ & NÃO & SIM \\
\hline ARGILOSO & $\begin{array}{l}\text { CANA-DE- } \\
\text { AÇÚCAR }\end{array}$ & CONVENCIONAL & $\begin{array}{c}\text { VITAVAX THIRAM } 200 \\
\text { SC }\end{array}$ & NÃO & SIM \\
\hline ARGILOSO & AMENDOIM & CONVENCIONAL & $\begin{array}{c}\text { VITAVAX THIRAM } 200 \\
\text { SC }\end{array}$ & NÃO & SIM \\
\hline ARGILOSO & $\begin{array}{l}\text { CANA-DE- } \\
\text { AÇÚCAR }\end{array}$ & CONVENCIONAL & $\begin{array}{c}\text { VITAVAX THIRAM } 200 \\
\text { SC }\end{array}$ & SIM & SIM \\
\hline ARENOSO & $\begin{array}{l}\text { CANA-DE- } \\
\text { AÇÚCAR }\end{array}$ & CONVENCIONAL & $\begin{array}{c}\text { VITAVAX THIRAM } 200 \\
\text { SC }\end{array}$ & SIM & SIM \\
\hline ARENOSO & $\begin{array}{l}\text { CANA-DE- } \\
\text { AÇÚCAR }\end{array}$ & CONVENCIONAL & $\begin{array}{c}\text { VITAVAX THIRAM } 200 \\
\text { SC }\end{array}$ & NÃO & SIM \\
\hline ARENOSO & AMENDOIM & CONVENCIONAL & $\begin{array}{c}\text { VITAVAX THIRAM } 200 \\
\text { SC }\end{array}$ & SIM & SIM \\
\hline ARENOSO & $\begin{array}{l}\text { CANA-DE- } \\
\text { AÇÚCAR }\end{array}$ & CONVENCIONAL & $\begin{array}{c}\text { VITAVAX THIRAM } 200 \\
\text { SC }\end{array}$ & SIM & SIM \\
\hline ARENOSO & $\begin{array}{l}\text { CANA-DE- } \\
\text { AÇÚCAR }\end{array}$ & CONVENCIONAL & $\begin{array}{c}\text { VITAVAX THIRAM } 200 \\
\text { SC }\end{array}$ & NÃO & SIM \\
\hline ARENOSO & POUSIO & $\begin{array}{c}\text { CONVENCIONAL E } \\
\text { MEIOSE }\end{array}$ & $\begin{array}{c}\text { VITAVAX THIRAM } 200 \\
\text { SC }\end{array}$ & NÃO & SIM \\
\hline ARENOSO & POUSIO & $\begin{array}{c}\text { CONVENCIONAL E } \\
\text { MEIOSE }\end{array}$ & $\begin{array}{c}\text { VITAVAX THIRAM } 200 \\
\text { SC }\end{array}$ & NÃO & SIM \\
\hline
\end{tabular}

O desenvolvimento do banco de dados e do sistema web seguiu o processo padrão de desenvolvimento de software da Embrapa. O Sistema Gerenciador de Bancos de Dados (SGBD) utilizado foi o PostgreSQL com extensão espacial PostGIS (PostGIS, 2020). Complementarmente, foi utilizado o shapefile do polígono dos municípios brasileiros disponível no site do IBGE (IBGE, 2020). Para apresentação dos dados foi desenvolvido um sistema de informação Web na linguagem de programação Java que, a partir do servidor de mapas GeoServer (GEOSERVER, 2020), mapeia 
todos os locais de origem das amostras recebidas, especificando a quantidade de amostras analisadas. Visando assegurar a confidencialidade relativa à origem das amostras, as informações disponibilizadas publicamente foram compiladas considerando o número total de amostras por município e por safra, as quais foram representas por círculos relativos ao número total de amostras analisadas por município, sendo o tamanho dos círculos determinados pelos intervalos: 0-5; 6-10; 11-20; 21-30; 31-40; 41-50; 51-100; >100. Para representar a presença ou ausência do patógeno optou-se por adotar um sistema do tipo "heat-colors" variando de azul (ausência do patógeno) a vermelho (patógeno presente em mais de $5 \%$ das amostras analisadas). Para garantir a integridade de domínio no banco de dados "Radar - Carvão do Amendoim", foram modeladas tabelas de domínio com o conjunto de valores possíveis para os campos sistema de cultivo, variedade, tipo de solo, cultivo e categoria da semente.

\section{Resultados e discussão}

Nós desenvolvemos um banco de dados, aliado a uma interface pública, que permite o acompanhamento da distribuição do agente causal do carvão do amendoim em áreas de plantios comerciais no Brasil. $O$ presente banco de dados e sua interface pública foram desenvolvidos usando ferramentas de livre acesso e fácil configuração, de modo a não criar custos de aquisição e manutenção, bem como dependência tecnológica. Optou-se pelo GeoServer, como servidor de mapas, pelo fato do mesmo possuir um sistema cartográfico com diversas funcionalidades adicionais (GEOSERVER, 2020). Semelhantemente, o PostgreSQL foi escolhido por ser o banco de dados livre mais avançado do mundo, exemplo para a especificação ANSI-SQL, e com ricas extensões para armazenar e tratar dados espaciais (POSTGRESQL, 2020).

Buscando a eficácia no fornecimento das informações publicamente disponibilizadas no mapa, utilizou-se as variáveis visuais forma, tamanho e cor, implantadas em localizações pontuais (ARCHELA, TÉRRY, 2008). A forma foi o círculo em tamanhos proporcionais aos valores que cada município apresenta em relação ao número de amostras 
analisadas e a cor representando o percentual de amostras contaminadas (Figura 1).

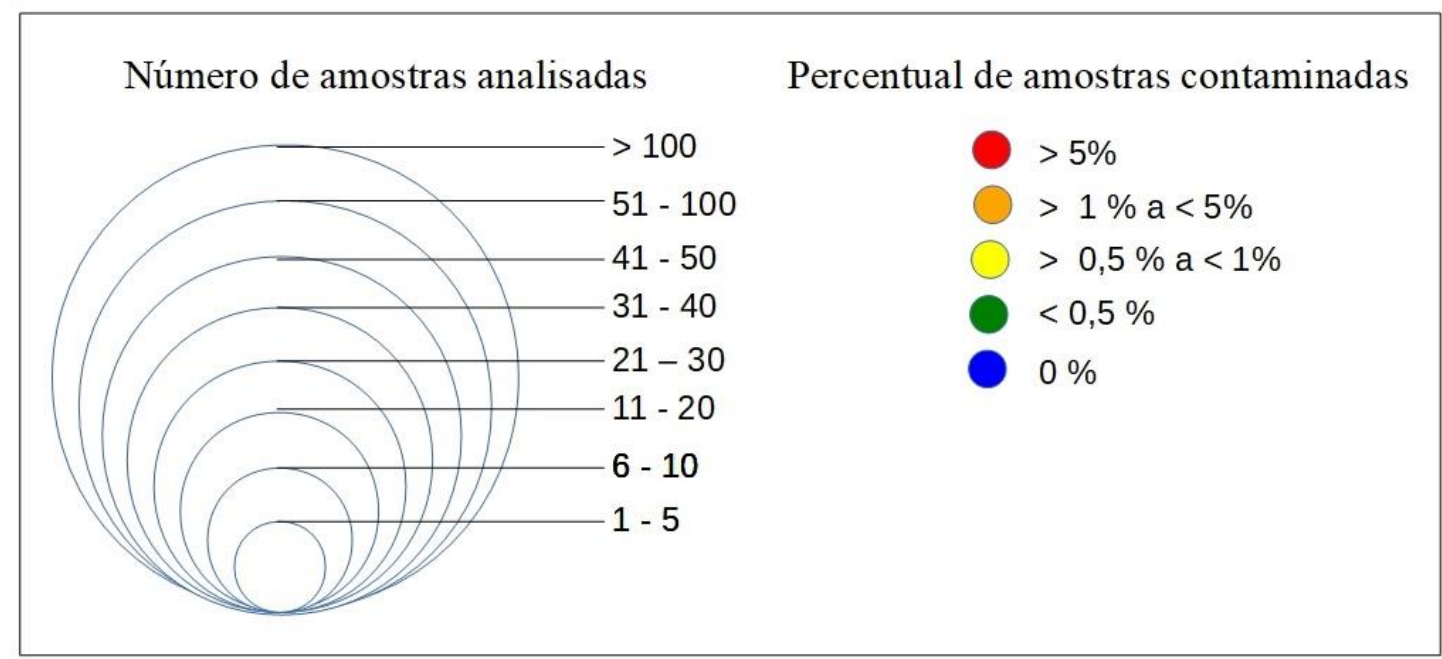

Figura 1. Legenda das informações apresentadas no mapa interativo do Radar - Carvão do Amendoim.

O uso desse tipo de indicador visual permite que a informação a ser transmitida seja de fácil compreensão e prontamente assimilada pelo público alvo. Nesse sentido, a interface pública do Radar - Carvão do Amendoim, permite ao usuário a rápida identificação da origem das amostras (representada por cada círculo), da representatividade das mesmas (representada pelo tamanho do círculo) e da presença ou não do agente causal do carvão do amendoim (representada pela cor do círculo). Adicionalmente, ao se passar o cursor do mouse, sobre cada um dos círculos, uma janela pop-up é apresentada detalhando o número de amostras analisadas para cada safra (Figura 2). 


\section{Embrapa Radar: Carvão do Amendoim}

Distribuição de amostras analisadas

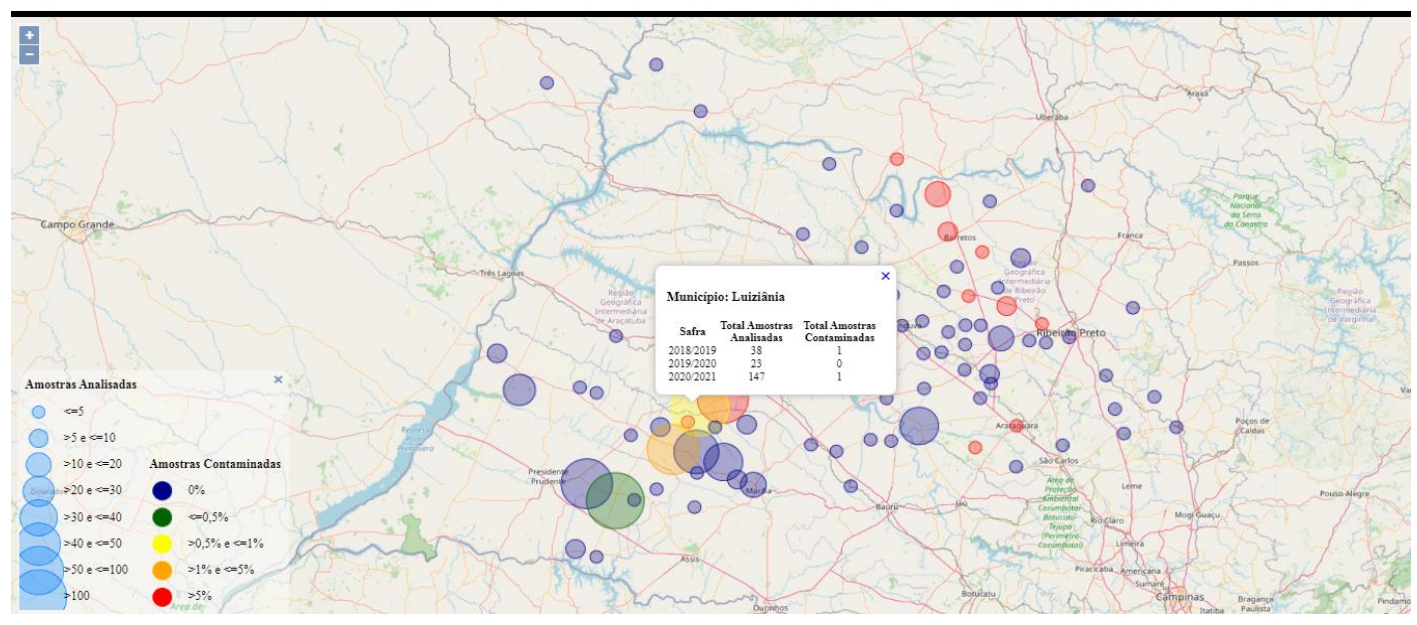

Figura 2. Exemplo da interface pública do "Radar - Carvão do Amendoim", destacando a janela pop-up, visualizada ao se passar o mouse sobre cada círculo, evidenciando o nome do município, o número de amostras por safra e o número de amostras contaminadas (os dados apresentados são fictícios e foram utilizados apenas para fins de ilustração).

Mapas de distribuição da ocorrência de pragas são utilizados em diversas situações, mas são especialmente utilizados nos casos de pragas de elevado risco epidêmico. Cazón et al. (2018) apresentaram um mapa da Província de Córdoba, Argentina, mostrando o gradiente estático da incidência do carvão do amendoim com base na amostragem de apenas 30 lotes comerciais de amendoim. Embora essa abordagem seja útil, devido a dinâmica da doença, e especialmente pelo fato de que no estado de São Paulo, a cultura do amendoim pode ser considerada uma cultura itinerante, nós concluímos que seria necessária uma forma que permitisse um acompanhamento mais dinâmico da distribuição do patógeno. Com o desenvolvimento do presente banco de dados e sua interface pública será possível acompanhar anualmente a situação das principais áreas produtoras de amendoim, quanto a presença ou não do patógeno. Embora o banco de dados tenha sido desenvolvido para mapear as áreas de produção de amendoim do Brasil, a forma como o mesmo foi construído permite o mapeamento do patógeno em qualquer local, podendo vir a ser uma ferramenta útil para mapear a distribuição do patógeno em outros países, em especial na Argentina. 
Apenas na safra 2018/2019 foram recebidas mais de 630 amostras, das quais cerca de 50 não continham as informações mínimas que permitissem sua rastreabilidade (origem geográfica), de forma que não foram incluídas no banco de dados. Atualmente o banco conta com aproximadamente 580 amostras, provenientes de mais de 80 municípios do estado de São Paulo e circunvizinhos. Após a liberação de acesso, o "Radar - Carvão do Amendoim" poderá ser feito a partir do endereço: http://www.cnpa.embrapa.br/rca.

\section{Conclusões}

O banco de dados "Radar - Carvão do Amendoim" foi desenvolvido com sucesso a partir do conjunto de informações obtidas e, sua interface pública, permite visualizar a distribuição das amostras recebidas e analisadas a cada safra. A constante alimentação do mesmo será crucial para identificar focos iniciais da ocorrência do patógeno e, a partir dessa identificação, acompanhar sua dispersão ou retração ao longo dos anos, subsidiando às autoridades sanitárias para tomada de decisão e provendo a pesquisa com dados diversificados que auxiliarão no melhor entendimento da epidemiologia da doença e, por consequência, na definição das melhores estratégias de combate a mesma.

\section{Agradecimentos}

Esse estudo só foi possível graças a colaboração das empresas e cooperativas produtoras e beneficiadoras de amendoim do estado de São Paulo. Os autores também gostariam de agradecer a Câmara Setorial do Amendoim do Estado de São Paulo pelo constante apoio na realização do presente estudo. Marcel N. B. Sugimoto, particularmente, agradece também ao CNPq pela bolsa de PIBIC. 


\section{Referências}

ARCHELA, Rosely Sampaio; THÉRY, Hervé. Orientação metodológica para construção e leitura de mapas temáticos, Confins [Online]. v.3, n.3, p.1-21, 2008. DOI: https://doi.org/10.4000/confins.3483

CAZÓN, Luis Ignacio et al. The biology of Thecaphora frezii smut and its effects on Argentine peanut production. In: KIMATU, Josphert Ngui (ed). Advances in Plant Pathology. Rijeka, InTech Publishing, 2019. p.31-46. http://dx.doi.org/10.5772/intechopen.75837

GEOSERVER. GeoServer - open source server for sharing geospatial data. Disponível em http://geoserver.org. Acesso em: 30 de jun. 2020.

IBGE. Instituto Brasileiro de Geografia e Estatística. Disponível em http://geoservicos.ibge.gov.br/geoserver/wms?service=WFS\&version=1.0.0 \&request=GetFeature\&typeName=CCAR:BC250_Municipio_A\&outputForm at=SHAPE-ZIP. Acesso em: 30 de jun. 2020.

PostGIS. PostGIS - Spatial and Geographic objects for PostgreSQL. Disponível em https://postgis.net/. Acesso em: 30 de jun. 2020.

PostgreSQL. PostgreSQL - The World's Most Advanced Open Source Relational Database. Disponível em https://www.postgresql.org/. Acesso em: 30 de jun. 2020.

RAGO, Alejandro Mario, et al. Peanut smut: from an emerging disease to an actual threat to Argentine peanut production. Plant Disease, v. 101, n. 3, p. 400-408, 2017. https://doi.org/10.1094/PDIS-09-16-1248-FE

SOARES, Dartanhã José. Alerta - carvão do amendoim: uma potencial ameaça! Empresa Brasileira de Pesquisa Agropecuária. 2018. Disponível em: http://www.infoteca.cnptia.embrapa.br/infoteca/handle/doc/1094108. Acesso em: 30 de jun. 2020.

SOARES, Dartanhã José. Carvão do amendoim: uma ameaça no ar e nas sementes. In: SILVA, Rouverson Pereira da et al. (ed.) Avanços na produção de amendoim. Jaboticabal: Funep, 2019. p.113-124.

SUGIMOTO, Marcel Norie Bergamin; ZAGO, Lucas Fernando Alvez; SOARES, Dartanhã Jose. Monitoramento da ocorrência de Thecaphora frezzii em amostras de amendoim oriundas do Brasil. In.: LIMA, Marleide Magalhães de Andrade et al. (ed.). Resumos da XIV Encontro de Produção Científica da Embrapa Algodão, Campina Grande, PB, 05 de setembro de 2019. - Brasília, DF: Embrapa, 2019. p. 15. 